criticized by Saitou and Omoto. Rather, we published a tree relating mtDNA molecules to one another. Molecular trees can be reliable if many sites are compared, as was the case in our study of 370 sites in each mtDNA. This molecular tree allowed us to trace all known human mtDNAs back to one mother who probably lived in Africa about 200,000 years ago ${ }^{2}$.

To cast doubt on this hypothesis, Saitou and Omoto ${ }^{1}$ built a population tree using genetic distances taken from our paper. The significance of their tree, which does not support an African origin, must be questioned for two reasons. First, the sample sizes were too small for accurate estimation of genetic distances. Second, instead of using the actual data (in Fig. 3 of ref. 2) they used the genetic distances in Table 1, which had been rounded off to one significant figure. The use of the rounded off numbers heightened the uncertainty in their tree.

We have increased the number of New Guineans sampled from 26 in the original article $^{2}$ to 55 (ref. 3) and then to 119 (ref. 4). Genetic distances between New Guineans and other populations are now more accurate. The resulting population tree shows that the African population is most divergent from other populations, whereas the New Guinea population is related most closely to that of Asia, as Stoneking et al. have noted ${ }^{3}$. This population tree contradicts the main claim of Saitou and Omoto' and supports the proposed African origin for human mtDNAs ${ }^{2}$.

As for their criticism of our mitochondrial time scale for human evolution, Saitou and Omoto' display no awareness of the primary paper on temporal calibration ${ }^{3}$, even though it was cited fully and explicitly?. They also misinterpret our estimate of the mean rate of divergence of human mtDNA. The value we proposed, $2-4 \%$ per million years, refers to the comparison of two lineages, not as they supposed to evolution along one lineage. Therefore, their criticisms of the time scale and its implications lack substance.

Finally, we are puzzled by Saitou and Omoto's claim' that, according to Cann et al. ${ }^{2}$, the African population diverged from other populations 200,000 years ago. We intentionally made no statement about times of divergence between populations because the concept of such a time is nearly meaningless for populations that still exchange genes. Perhaps our critics have not broken free from the constraints imposed by thinking in terms of gene frequencies, genetic distances, population trees, and time of divergence between populations. Studies of mtDNA have introduced a new way of looking at evolution below the species level ${ }^{5.6}$.

Darlu and Tassy ${ }^{7}$, too, generate confusion when they criticize molecular trees as if they were population trees. Nevertheless, their main point is valid. Because the molecular trees ${ }^{2}$ for mtDNA maps and D-loop sequences were rooted by the midpoint method, it cannot be certain that the mitochondrial mother of us all was African. For this reason, we merely stated that "Africa is a likely source". The case for an African origin, however, is not as weak as Darlu and Tassy ${ }^{7}$ claim. Their criticism neglects two justifications for the mid-point root, both of which foster the view that human mtDNA evolution is clock-like.

First, most $(70 \%)$ of the surviving point-mutational differences found among human mtDNAs seem to be neutral ${ }^{8}$. Hence, their accumulation is expected to be mainly a function of time. Because, according to three separate studies $^{2.9,10}$, the African population is more variable than any other human population tested, it is likely to be the oldest. Second, the observed rates at which point mutations accumulate on surviving mtDNA lineages are alike in the three human populations subjected to rate tests ${ }^{3}$, namely aboriginal New Guineans, Australians and Americans. Furthermore these rates are not lower than in other mammals $s^{5,11}$ and birds ${ }^{12}$. Such findings give empirical support for mid-point rooting.

Johnson et al. ${ }^{9}$ have made an ad hoc argument that the evolutionary rate may be higher on the primary branch leading exclusively to African mtDNAs, types $1-7$ in the tree for mtDNA maps ${ }^{2}$. If this were so, the midpoint method would falsely put the root on the African side of the true root. Although current data do not rule out an African acceleration, we should be aware that this argument has a corollary, which is that the average rate of human mtDNA evolution would be higher than we estimated ${ }^{2.3}$. As a consequence, our previous estimate of 200,000 years ago for the time when the common mitochondrial mother lived ${ }^{2 .}$ would have to be revised toward an even more recent time.

Department of Genetics,

Rebecca L. CANN

University of Hawaii,

Honolulu, Hawaii 96822, USA

MARK STONEKING

Department of Biochemistry,

University of California,

Berkeley, California 94720, USA

1. Saitou, N. \& Omoto, K. Nature 327, 288 (1987)

. Cann. R.L. et al. Nature 325, 31-36 (1987).

3. Stoneking, M. et al. Cold Spring Harb. Symp. quant. Biol. 51, 433-439(1986)

4. Stoneking, M. thesis, Univ. California (1986).

5. Wilson, A.C. et al. Biol. J. Linn. Soc. Lond. 26, 375-400 (1985)

6. Avise, J.C. Phil. Trans. R. Soc. B312, 325-342 (1986)

7. Darlu, P. \& Tassy P. Nature 329, 112 (1987)

8. Whittam, T.S. et al. Proc. natn. Acad. Sci. U.S.A. 83 , 9611-9615 (1986)

9. Johnson, M.J. et al. J. molec. Evol. 19, 255-27! (1983).

10. Horai, S., Gojobori, \& Matsunaga, E. Jap. J. Genet. 61, $271-275(1986)$

11. Higuchi, R.G.et al. J. molec. Evol. 25, 283-287 (1987).

12. Shields, G.F. \& Wilson, A.C. J. molec. Evol. 24, 212-217 (1987).

\section{Receptor-gene sequence}

SIR-In their paper giving the partial genomic sequence of $\mathrm{V} \alpha 112-2$ (part of the gene coding for the $\alpha$-chain of a mouse T-cell receptor) ${ }^{i}$, Hochgeschwender et al. located the $3^{\prime}$-end of an intron in an extremely unlikely place. Preceeding the canonical $\mathrm{AG}$ at the splice site is a run of 15 nucleotides of which 8 consecutive ones are purine nucleotides and only 4 are pyrimidine nucleotides. It is well established ${ }^{2}$ that purines are few and generally far between in this situation in other vertebrate introns. A little further upstream there is a sequence $\mathrm{Py}_{28}$ GCACTGTAG which is much more likely to be the $3^{\prime}$-end of an intron. If this splice acceptor site were used, the reading frame of the presumed RNA would still be preserved and there would be an insertion of five amino acids in the protein coded for (Gly-ArgThr-His-Gly-) preceeding the Asp residue as shown in Fig. 4 of Hochgeschwender et al. . The Gly-Asp linkage that would occur in this putative protein is a likely linkage for cleavage by the signal peptidase to produce the mature protein.

Department of Biochemistry,

JOHN D. HAWKINS

The Medical College of St Bartholomew's Hospital,

Charterhouse Square,

London EC1M $6 B Q, U K$

1. Hochgeschwender. U. et al. Nature 326, 307-309. (1987) 2. Mount, S. M. Nucleir Acids Res. 10. $459-469 .(1981)$

\section{Correction needed}

SIR-In my reply (Nature 328,$675 ; 1987$ ) to Collett and Loudon (Nature 326, 671; 1987), lines 4-6 at the top of column three should have read: . . . that my prediction remains tenable but that the prediction of the Copenhagen interpretation no longer clashes with mine (as it does in the case of a "fixed source"). Unfortunately you omitted the words now put in italics.

If you allow me to add a comment on Collett and Loudon's reply that follows my letter, I would point out that in their original criticism they speak of a "fixed source", whereas in their new criticism they replace this by a "massive source". To my mind this means a change of the problem: they never explain why a (nonmassive positronium) source cannot be "fixed".

136 Welcomes Road,

KARL PoPPER

Kenley, Surrey CR2 $5 H H, U K$

\section{Scientific Correspondence}

Scientific Correspondence is intended to provide a forum in which readers may raise points of a scientific character. They need not arise out of anything published in Nature. In any case, priority will be given to letters of less than 500 words and five references. 